\title{
Carrier Transport Properties of GAN in High Electric Field
}

\author{
Pei Ling Cheang, Eng Kiong Wong, Lay Lian Teo
}

\begin{abstract}
The Monte Carlo (MC) simulation of the carrier transport mechanisms including impact ionization at high electric field in GaN is presented. Two non-parabolic conduction and valence bands were considered for the simulation of transport properties of electron and hole respectively. The carriers' drift velocity and energy are simulated as a function of applied electric field at room temperature. The maximum velocity of electron is $2.85 \times 10^{7} \mathrm{~cm} / \mathrm{s}$ at $140 \mathrm{kV} / \mathrm{cm}$. The velocity of electron is saturated at $2 \times 10^{7} \mathrm{~cm} / \mathrm{s}$ at electric field greater than $300 \mathrm{kV} / \mathrm{cm}$. In our work, the velocity of hole is $5 \times 10^{6} \mathrm{~cm} / \mathrm{s}$ at $500 \mathrm{kV} / \mathrm{cm}$. Electron energy increases as the electric field increase and fluctuated at electric field greater than $600 \mathrm{kV} / \mathrm{cm}$ when impact ionization occurred. The impact ionization rates are obtained by using modified Keldysh equation. The hole impact ionization rate is higher than that of electron. This work also shows higher electron impact ionization coefficient than that of hole at electric field greater than $4.04 \mathrm{MV} / \mathrm{cm}$.
\end{abstract}

Index Terms: drift velocity, Gallium nitride, impact ionization, Monte Carlo.

\section{INTRODUCTION}

Ultraviolet (UV) radiation, though occupy small portion of the total solar radiation and absorbed mostly by ozone in the stratosphere before landing on the surface of the earth, has effects on human life with application ranges from civil, industrial, military, environmental and biological sectors. In the past few decades, UV avalanche photodiodes (APDs) have been used to trace leakage, monitor solar UV, sense ozone and in advanced communications [1,2]. The stability in hot environments enable them to detect flame in harsh environments and missiles for military. Their wide range applications have caught significant attention and attempts to fabricate and optimize UV APDs to greater sensitivity, better signal-to-noise ratio, wider spectral selectivity and fast response besides thermally stable. Nano technologies nowadays have moved UV APDs towards higher precision and lower energy consumption besides miniaturization.

Detecting UV requires material to possess wide band gap that show strong absorption and sharp cut-off wavelength in the UV through near infrared spectral range to capture UV radiation that is low in intensity. Gallium nitride $(\mathrm{GaN})$ with energy gap of $3.4 \mathrm{eV}$ meets the spectral selectivity by offering high sensitivity in both visible-blind and solar-blind UV detections [3-5]. Its direct-bandgap offers higher quantum

Revised Manuscript Received on August 18, 2019

Pei Ling Cheang, Faculty of Engineering and Technology, Multimedia University, Melaka, Malaysia.

Eng Kiong Wong, Faculty of Engineering and Technology, Multimedia University, Melaka, Malaysia.

Lay Lian Teo, Faculty of Engineering and Technology, Multimedia University, Melaka, Malaysia. efficiency and consequently high gains. However, the nearly closed of the hole- and electron impact ionization coefficients of GaN cause it to have higher excess noise and hence significant dark current near breakdown at high reverse bias voltage in APDs. To achieve high multiplication gain and low excess noise factor, one of the ionization coefficients should be much larger than the other or the carrier with higher ionization coefficient should initiate the multiplication $[6,7]$. Thus, both hole and electron impact ionization coefficients determine the performance of GaN APDs. Oguzman et al. [8] reported the first theoretical study of hole initiated impact ionization in the zinc-blende and wurtzite phases of $\mathrm{GaN}$ by using Monte Carlo simulations. They concluded that the hole impact ionization coefficient is significantly higher than that of electron at electric field below $3.7 \mathrm{MV} / \mathrm{cm}$ in the wurtzite phase. Chen et al. [9] studied the impact ionization process by using ensemble Monte Carlo (EMC) method, had simulated the electron impact ionization coefficient higher than that of hole at electric field greater than $3.8 \mathrm{MV} / \mathrm{cm}$. In contrast, Bertazzi et al. [10] reported electron ionization coefficient greater than hole for electric field below $4 \mathrm{MV} / \mathrm{cm}$ along $\Gamma-\mathrm{M}$ direction in wurtzite GaN. Their simulation results showed that hole-initiated multiplication dominated in $\Gamma-\mathrm{A}$ direction for electric field above $3.2 \mathrm{MV} / \mathrm{cm}$.

Much efforts had been reported in literatures in the investigation of the multiplication gain and excess noise factor of GaN but less attention was paid to the transport properties [11] such as drift velocity, energy and impact ionization coefficient of carriers, in particular of holes in GaN which are the fundamental factors that affect the performance of GaN APDs. Thus in this work, Monte Carlo method is employed to develop a model to simulate the transport properties such as drift velocity, energy, occupancy, ionization rates and ionization coefficients of holes and electrons in $\mathrm{GaN}$ under electric field.

\section{MONTE CARLO SIMULATION}

APDs are strongly reverse biased semiconductor-based photodetectors that operate at high electric fields to detect weak light, amplify it internally and convert it to electric current that is detectable by the electronics following them. When an APD is exposed to light, incident photon is absorbed by the intrinsic layer of the material atoms and produce an electron-hole pair. As the electric field of the depletion region increases as a result of increasing applied voltage, the drift velocity and kinetic energy of charge carriers increase. Should an electron (or a hole) achieves sufficient energy to break a bond when impacting with lattice atoms at any time

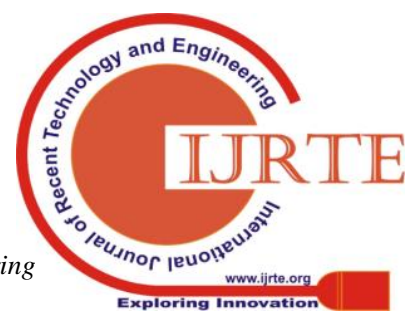


during the process, it generates a second electron-hole pair by impact ionization [12]. Impact ionization can either be initiated by an electron or a hole. Both the original carrier (electron or hole) and the generated electron-hole pair will then be accelerated by the electric field and has chances to further impact ionize and generate more electron-hole pairs, results in a chain reaction of avalanche multiplication that progressively increases the total number of carriers [13] and give an internal gain in the avalanche multiplication region. The process repeats until all electrons or holes leave the avalanche region.

As impact ionization is stochastic in nature, Monte Carlo (MC) method was employed in this work. Two non-parabolic conduction (valence) bands were considered for the simulation of electron (hole) and transport properties. The simulation began with the selection of an arbitrary wave vector, $\vec{k}_{x}$ as the initial condition of carrier. During the free flight, the carrier was accelerated in $k$-space by the conservation of momentum relation via

$k_{f}=k_{i}+\frac{e F t}{\hbar}$

where $k_{\mathrm{i}}$ and $k_{\mathrm{f}}$ are initial and final wave vectors respectively, $F$ is the applied electric field. Duration of flight time in each iteration is determined by

$\tau=-\frac{\ln r}{\Gamma_{\mathrm{o}}}$

where $r$ is a random number representing probability per unit time of a carrier during the flight that depends on the initial scattering rate $Г$ o. The flight time determined was used to calculate the velocity and energy of carriers during drift process. The total flight time must be long enough so that the initial condition has little influence on the final results. After the free flight, carrier with energy greater than the energy gap would scatter. Wave vector that was updated during the drift process was transferred to the scattering process. The scattering mechanism for carrier was chosen. By using Fermi Golden's Rule, scattering mechanisms considered are acoustic phonon, optical phonon, polar optical phonon and impurity scattering using the parameters listed in Table 1. Following that, the carrier's state and its wave vector after scattering were identified. The simulation process repeated to evaluate the drift motion under constant electric field up to $1000 \mathrm{kV} / \mathrm{cm}$ and the following scattering process until the maximum flight time was achieved.

The transport properties of GaN were reported by several researchers [9-11, 14-18]. Carrier's drift velocity and average energy during the total simulation time $t_{\max }$ were simulated respectively using

$$
\vec{v}=\frac{\sum\left(E_{f}-E_{i}\right)}{q \overrightarrow{F_{x}} t_{\max }}
$$

and

$E=\frac{1}{t_{\max }} \sum\left(\frac{E_{i}+E_{f}}{2}\right) \tau$ where $q$ is the charge of carrier, $E_{i}$ is carrier's energy at the beginning of the flight and $E_{f}$ is carrier's energy at the end of the flight.

Table 1. Material Parameters of Wurzite GaN

\begin{tabular}{|c|c|c|}
\hline Material & GaN & \\
\hline Types of Carrier & Electron & Hole \\
\hline Energy band gap $(\mathrm{eV})$ at $300 \mathrm{~K}$ & 3.42 & 3.42 \\
\hline $\begin{array}{l}\text { Effective masses }\left(m_{\mathrm{o}}\right) \\
\text { - First band } \\
\text { - Second band }\end{array}$ & $\begin{array}{l}0.2 \\
1.5\end{array}$ & $\begin{array}{l}1.4 \\
0.3\end{array}$ \\
\hline Sound velocity $\left(\mathrm{cm} \mathrm{s}^{-1}\right)$ & $7.96 \times 10^{5}$ & $4.13 \times 10^{5}$ \\
\hline $\begin{array}{l}\text { Dielectric constant: } \\
\text { - Static relative permittivity } \\
\left(\varepsilon_{0}\right) \\
\text { - Optical relative permittivity } \\
\left(\varepsilon_{\infty}\right)\end{array}$ & $\begin{array}{l}9.50 \\
5.35\end{array}$ & $\begin{array}{l}9.50 \\
5.35\end{array}$ \\
\hline Mass density $\left(\mathrm{g} \mathrm{cm}^{-3}\right)$ & 6.15 & 6.15 \\
\hline $\begin{array}{l}\text { Polar optical phonon energy } \\
(\mathrm{meV})\end{array}$ & 91.2 & 91.2 \\
\hline $\begin{array}{l}\text { Acoustic deformation potential } \\
(\mathrm{eV})\end{array}$ & 8.54 & 19.60 \\
\hline $\begin{array}{l}\text { Non-parabolicity constant } \\
\left(\mathrm{eV}^{-1}\right)\end{array}$ & 0.189 & - \\
\hline $\begin{array}{l}\text { Energy separation }(\Gamma \text { and } M-L \\
\text { valleys) }(\mathrm{eV})\end{array}$ & 1.9 & 0.011 \\
\hline $\begin{array}{l}\text { Energy separation }(\Gamma \text { and } A \\
\text { valleys })(e V)\end{array}$ & 1.3 & - \\
\hline
\end{tabular}

Impact ionization was considered as an additional scattering mechanism in this work. The impact ionization rates were modeled by using modified Keldysh equation as below.

$\frac{1}{\tau_{i i}^{j}(\varepsilon)}=P_{j}\left(\varepsilon-\varepsilon_{t h}^{j}\right)^{\gamma_{j}}$

where $j$ is a band index, $P_{j}$ is the softness coefficient, $\varepsilon_{t h}^{j}$ is the ionization threshold energy and $\gamma_{j}$ is the power exponent using the parameters listed in Table 2.

Table 2. The Parameters for Electron and Hole Impact Ionization Rates in $\mathrm{GaN}$

\begin{tabular}{|c|c|c|c|c|}
\hline Parameter & $i$ & $P_{j}$ & $\gamma_{j}$ & $\varepsilon_{t h}^{j}(\mathrm{eV})$ \\
\hline \multirow{2}{*}{ Electron } & 1 & $1.00 \times 10^{10}$ & 5 & 3.42 \\
\hline & 2 & $2.00 \times 10^{10}$ & 5 & 3.50 \\
\hline Hole & 1 & $6.00 \times 10^{10}$ & & \\
\hline
\end{tabular}


\begin{tabular}{l|l|l|l|l}
2 & $1.00 \times 10^{11}$ & 5 & 3.80
\end{tabular}

Impact ionization coefficients [18] were computed based on the number of times the electron $\left(n_{e}\right)$ or hole $\left(n_{h}\right)$ scattered due to impact ionization in the momentum space and the travelling distance of electron $\left(l_{e i}\right)$ and that of hole $\left(l_{h i}\right)$. The electron $(\alpha)$ and hole $(\beta)$ impact ionization coefficients are the reciprocal of averaging distance for these carriers respectively as following.

$\alpha=\left[\sum_{i=1}^{n_{e}} l_{e i} / n_{e}\right]^{-1} \mathrm{~cm}^{-1}$

$\beta=\left[\sum_{i=1}^{n_{h}} l_{h i} / n_{h}\right]^{-1} \mathrm{~cm}^{-1}$

\section{RESULTS AND DISCUSSION}

Monte Carlo method was applied in this work to simulate the carrier transport properties in GaN using the parameters shown in Table 1 The velocity and energy of electron and hole in $\mathrm{GaN}$ were simulated.

Fig. 1 shows the comparison of electron drift velocity of $\mathrm{GaN}$ under electric field with literature. At low field region, our result is closed to measurement of Ardaravicius et al. [14] at electric field between $70 \mathrm{kV} / \mathrm{cm}$ to $120 \mathrm{kV} / \mathrm{cm}$. Most of the measurement data in literature reported electron drift velocity at low electric field. The electron drift velocity in our work increases with the electric field and achieves a maximum value of $2.85 \times 10^{7} \mathrm{~cm} / \mathrm{s}$ at a critical field of $140 \mathrm{kV} / \mathrm{cm}$. As the electric field increases, the electrons gain more energy and are excited to the higher band where they become heavier. The possibility of their collisions increases and slows them. For the further increase in the electric field strength beyond $300 \mathrm{kV} / \mathrm{cm}$, the electron drift velocity saturated at $2.00 \times 10^{7}$ $\mathrm{cm} / \mathrm{s}$. It is noticed that our result is an average to most of the experimental and MC results [10-11, 14-17].

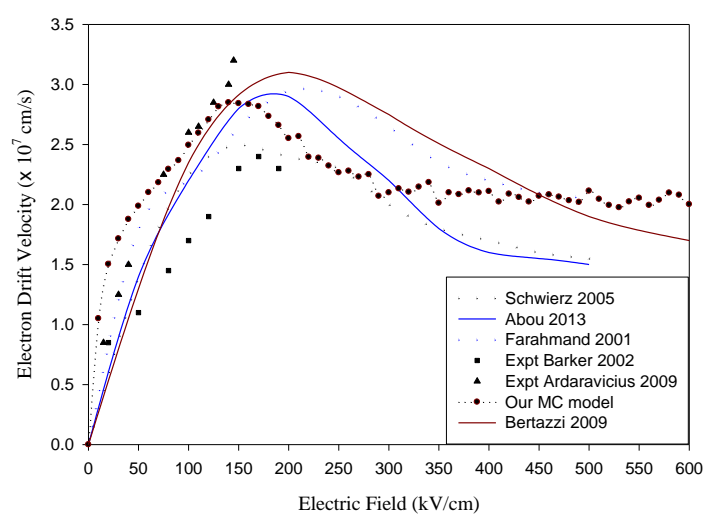

Fig. 1. Electron drift velocity of $\mathrm{GaN}$ as a function of electric field.

Fig. 2 shows the average electron energy of GaN as a function of electric field in our work. It is noticed that the average electron energy remains low and is only slightly higher than the thermal energy at electric field below 140 $\mathrm{kV} / \mathrm{cm}$. This is because electrons lost all the energy they obtained from electric field through polar optical phonon scattering at low field. There is a sudden increase in average electron energy around $140 \mathrm{kV} / \mathrm{cm}$. Beyond this electric field, the polar optical phonon scattering mechanism is unable to get rid of the whole energy gained from the electric field and other scattering mechanisms begin to take part. The average electron energy increases with the electric field and become fluctuates at electric field greater than $600 \mathrm{kV} / \mathrm{cm}$ where impact ionization occur. It is found that $5 \mathrm{eV}$ is the average electron energy when this happens.

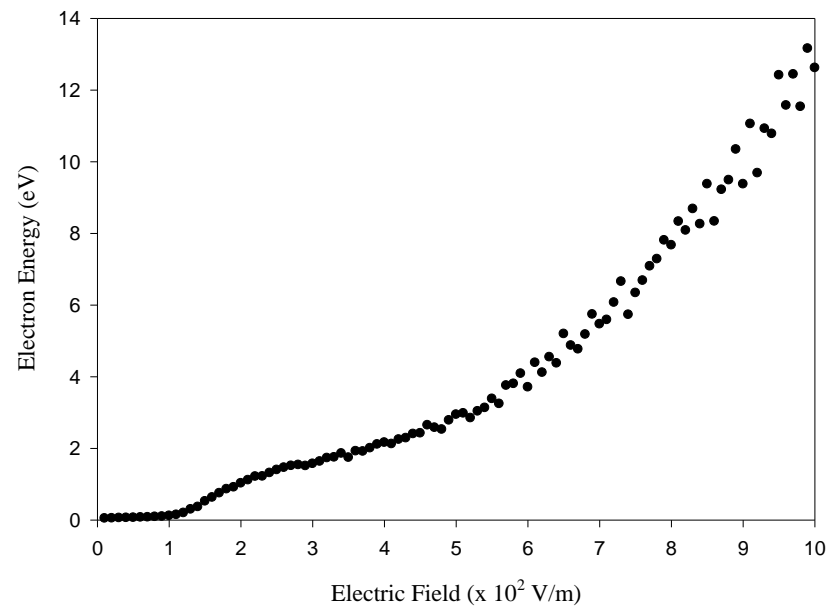

Fig. 2. Average electron energy of $\mathrm{GaN}$ as a function of electric field.

Fig. 3 shows the occupancy of electrons in $\mathrm{GaN}$ as a function of electric field in this work. The electrons occupy the first energy band when the electric field is below 140 $\mathrm{kV} / \mathrm{cm}$. Above $140 \mathrm{kV} / \mathrm{cm}$, the electrons gain sufficient energy from electric field and begin to be excited to higher energy band. The decrease of occupancy of electrons at first band to $40 \%$ and the increase to $60 \%$ at second band at electric field greater than $400 \mathrm{kV} / \mathrm{cm}$ shows that electrons are transferring to high energy band at high field $(>400 \mathrm{kV} / \mathrm{cm})$.

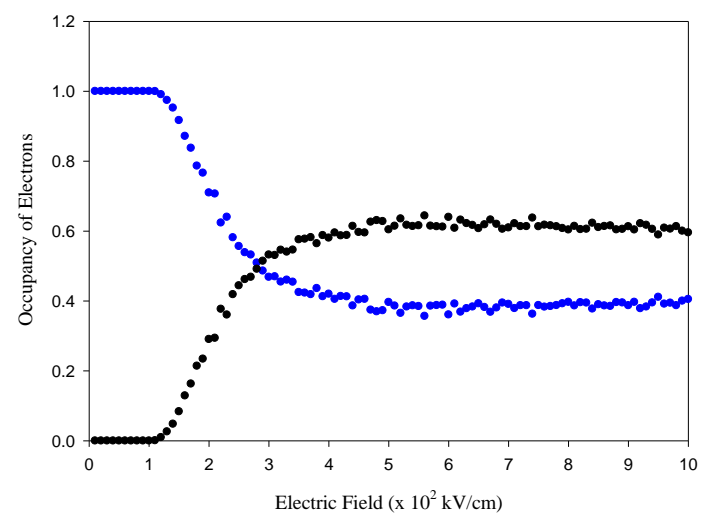

Fig. 3. Occupancy of electrons in $\mathrm{GaN}$ as a function of electric field. Blue dots: occupancy of electrons at first band. Black dots: occupancy of electrons at second band.

Fig. 4 shows the comparison of hole drift velocity simulation of $\mathrm{GaN}$ as a function of electric field with literature [9-10, 18]. Our results agree to Bertazzi et al. [10] at electric field less than $350 \mathrm{kV} / \mathrm{cm}$ and closed to Chen et al. [9] near 1 $\mathrm{MV} / \mathrm{cm}$. The drift velocity of holes is $5.0 \times 10^{6} \mathrm{~cm} / \mathrm{s}$ at 500 $\mathrm{kV} / \mathrm{cm}$ which is half of the electron velocity, $2.0 \times 10^{7} \mathrm{~cm} / \mathrm{s}$. The maximum drift velocity of holes achieved in this work is $7 \times 10^{6} \mathrm{~cm} / \mathrm{s}$ at $1 \mathrm{MV} / \mathrm{cm}$. So far there is no measurement data and very little MC model 
data for hole drift velocity available in the literature.

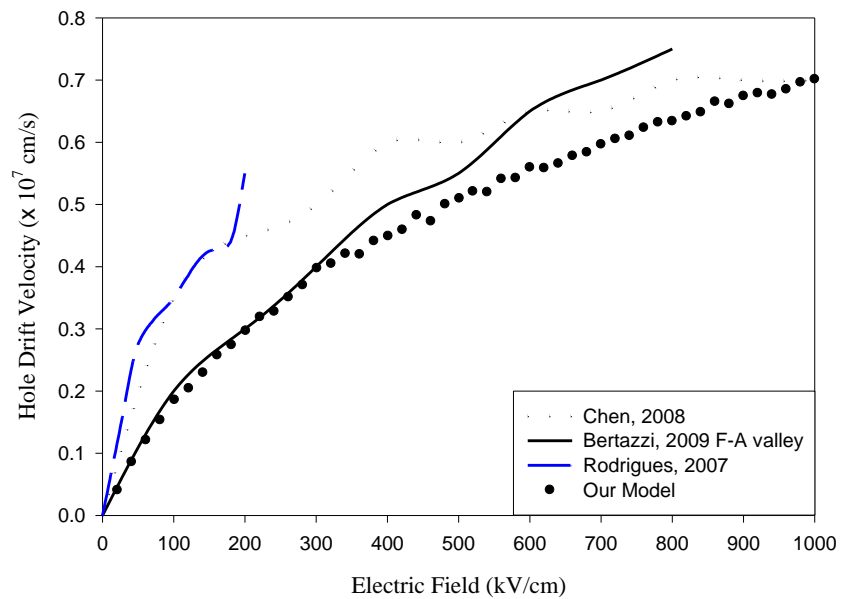

Fig. 4. Hole drift velocity of $\mathrm{GaN}$ as a function of electric field.

Fig. 5 shows the average hole energy of $\mathrm{GaN}$ as a function of electric field in our work. The average hole energy of GaN increases from $0.04 \mathrm{eV}$ to $0.12 \mathrm{eV}$ when electric field increases to $1 \mathrm{MV} / \mathrm{cm}$. The average hole energy is lower than the average electron energy.

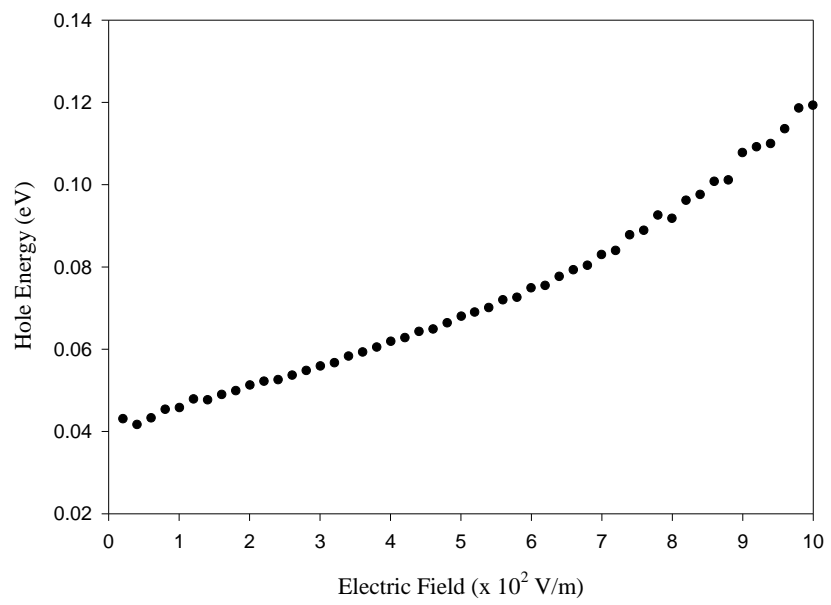

Fig. 5. Average hole energy of $\mathrm{GaN}$ as a function of electric field.

Fig. 6 shows the occupancy of holes in $\mathrm{GaN}$ as a function of electric field in this work. It is noticed that all holes reside on the heavy band because they are too heavy to move to light band.

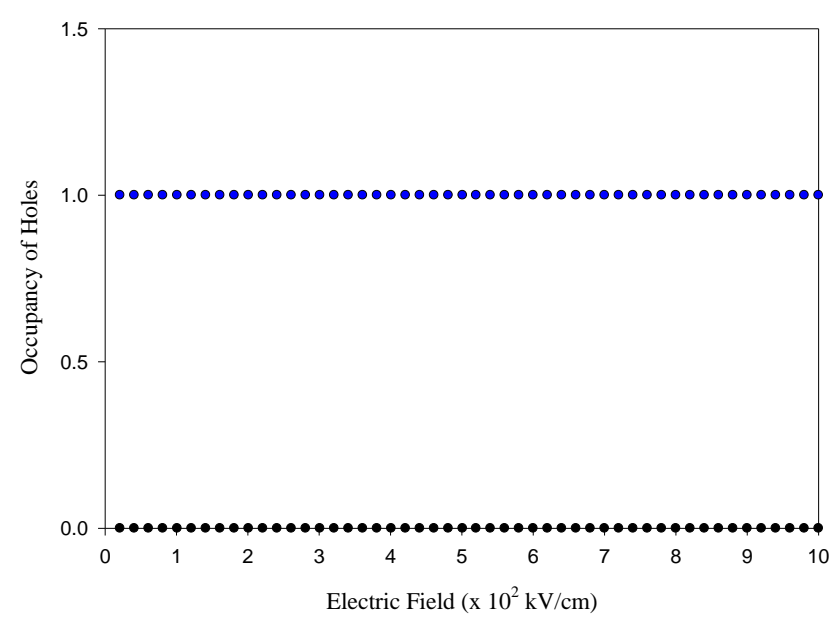

Fig. 6. Occupancy of holes in $\mathrm{GaN}$ as a function of electric field. Blue dots: occupancy of holes at first band. Black dots: occupancy of holes at second band.

Apart from the drift velocity and energy of carriers, the hole- and electron impact ionization rates were also simulated using modified Keldysh equation with parameters shown in Table 2. Fig. 7 and Fig. 8 show the impact ionization rate as a function of impacting carrier energy of electron and hole, respectively for $\mathrm{GaN}$ generated from our model, as compared to Bertazzi et al [10] and Oguzman et al. [8]. A power exponent of 5 is used in Keldysh formula to generate the impact ionization rate in first and second conduction bands in our model. It is shown that the impact ionization rate at higher energy band is considerably higher than the lower energy band due to the larger energy possessed by the carriers at the higher energy band. It can be clearly seen that hole impact ionization rate is higher than that of electron.

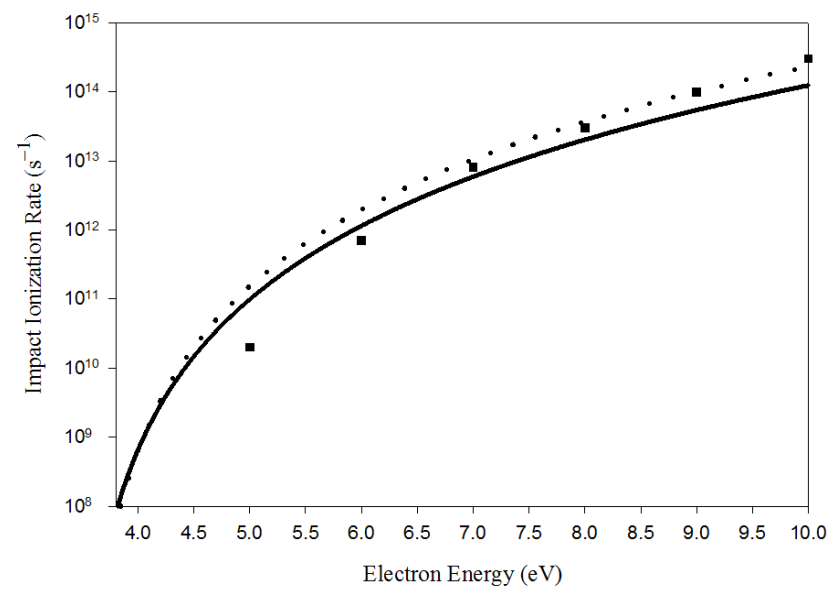

Fig. 7: The electron impact ionization rate as a function of impacting carrier energy for GaN. The solid and dashed lines are the ionization rates from the first and second conduction bands, respectively. The square represents the ionization rate by Bertazzi et al. [10]. 


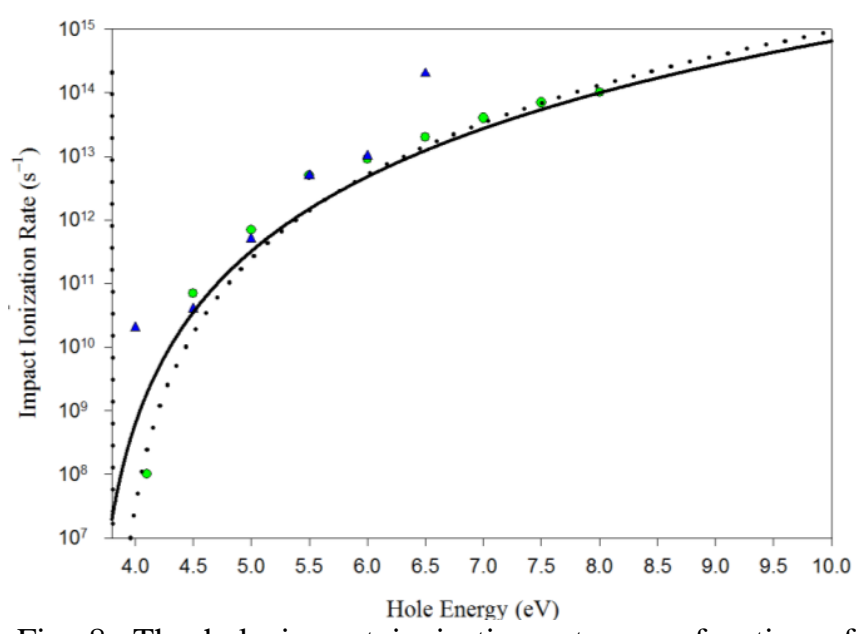

Fig. 8: The hole impact ionization rate as a function of impacting carrier energy for GaN. The solid and dashed lines are the ionization rates from the first and second valence bands, respectively. The circle represents the ionization rate by Bertazzi et al. [10] and triangle represents the ionization

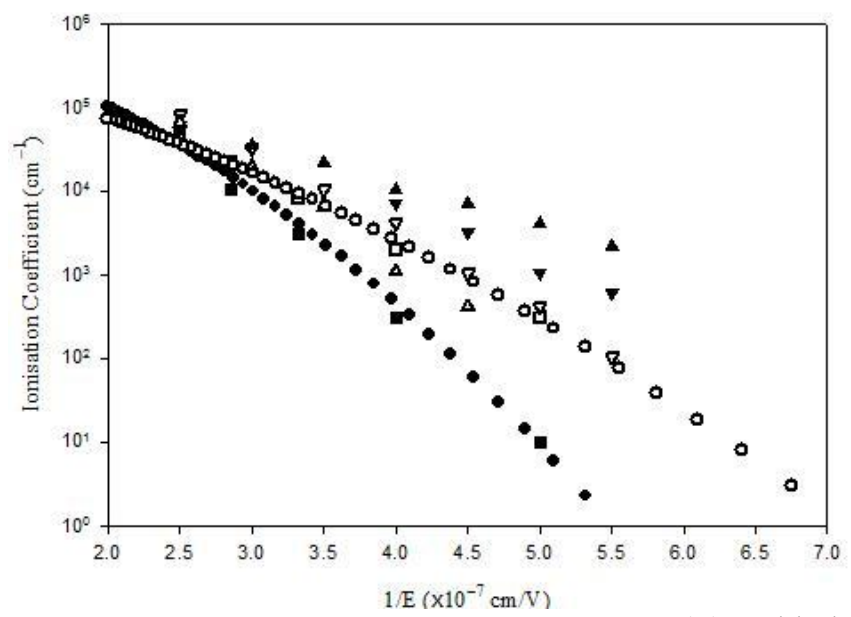

Fig. 9. Electron impact ionization coefficient, $\alpha(\bullet)$ and hole impact ionization coefficient, $\beta(\circ)$ of this work are compared with those by Oguzman et al. [8] ( $\alpha: \mathbf{m}, \beta$ : $\square)$ and Bertazzi et al. [10] ( $\alpha$ along $\Gamma$-M: $\boldsymbol{\Delta}, \beta$ along $\Gamma$-M: $\Delta ; \alpha$ along $\Gamma$-A: $\boldsymbol{\nabla}, \beta$ along $\Gamma-\mathrm{A}: \nabla$ ).

The $\alpha$ and $\beta$ which determine the performance of APDs were also simulated using parameters such as energy bandgap, effective masses of electron and hole shown in Table 1. Since the ionization behaviour of carriers is greatly influenced by the electric field, the electron and hole impact ionization coefficients were simulated as a function of inverse electric field by using (6) and (7) as shown in Fig. 9. It is shown that our electron impact ionization coefficient, $\alpha$ is slightly higher than that of hole, $\beta$ at electric field above 4.04 $\mathrm{MV} / \mathrm{cm}$, comparable at $4.04 \mathrm{MV} / \mathrm{cm}$ but diverge with $\beta$ become higher than $\alpha$ at electric field below $4.04 \mathrm{MV} / \mathrm{cm}$. The disparate of $\alpha$ and $\beta$ values are pivotal to bring about a low excess noise and high multiplication gain APD. Our higher $\beta$ as compared to $\alpha$ at electric field below $4.04 \mathrm{MV} / \mathrm{cm}$ closed to Oguzman et al. [8] work shows that the holes dominate the multiplication process at low field. Our $\beta$ also closed to Bertazzi et al. [10] along $\Gamma$-A direction even though their rate by Oguzman et al.[8].

simulated results showed the hole impact ionization is dominated at electric field above $3.2 \mathrm{MV} / \mathrm{cm}$. Based on the impact ionization coefficients curve plotted in Fig. 9, the electric field dependent impact ionization coefficient expressions obtained in this work are deduced as (8) and (9), where $E$ is the electric field.

$$
\begin{aligned}
& \alpha=7.32 \times 10^{7} \exp \left[-\left(\frac{7.16 \times 10^{8}}{E}\right)^{1.90}\right] \mathrm{cm}^{-1} \\
& \beta=3.48 \times 10^{7} \exp \left[-\left(\frac{6.56 \times 10^{8}}{E}\right)^{1.65}\right] \mathrm{cm}^{-1}
\end{aligned}
$$

\section{CONCLUSION}

In this work, Monte Carlo (MC) simulation is employed to investigate the carrier transport mechanisms including impact ionization at high electric field in $\mathrm{GaN}$ at $300 \mathrm{~K}$. Two non-parabolic conduction and valence bands were considered for the simulation of transport properties of electron and hole respectively. The simulated electron and hole drift velocities are consistent with the available literature. Both electron and hole energies increases with electric field with electron energy is higher than that of hole and fluctuated at electric field greater than $600 \mathrm{kV} / \mathrm{cm}$. The electrons in first band are excited to second band above $140 \mathrm{kV} / \mathrm{cm}$ while all holes reside on second band. The hole impact ionization rate is higher than that of electron. This work also shows higher electron impact ionization coefficient than that of hole at electric field greater than $4.04 \mathrm{MV} / \mathrm{cm}$.

\section{ACKNOWLEDGMENT}

This work is supported by FRGS/1/2017/TK04/MMU/03/5, MoHE

\section{REFERENCES}

1. E. Monroy, F. Omnès, and F. Calle, "Wide-bandgap semiconductor ultraviolet photodetectors," Semicond. Sci Technol. vol. 2 (4), R33, 2003.

2. Z. Alaie, S. M. Nejad, and M. H. Yousefi, "Recent advances in ultraviolet photodetectors," Mater. Sci. in Semicond. Process. vol. 29, pp. 16-55, 2015.

3. S. Verghese, K. McIntosh, R. Molnar, L. Mahoney, R. Aggarwal, M. Geis, K. Molvar, E. Duerr, and I. Melngailis, "GaN avalanche photodiodes operating in linear gain mode and Geiger mode," IEEE Trans. Electron. Devices. vol. 48 (3), pp. 502-511, 2001

4. R. Dupuis, J. H. Ryoua, S. C. Shena, P. D. Yoder, Y. Zhanga, H. J. Kima, S. Choia, and Z. Lochner, "Growth and fabrication of high-performance GaN-based ultraviolet avalanche photodiodes," J. Cryst. Growth. vol. 310 (23), pp. 5217-5222, 2008.

5. J. C. Carrano, D. J. H. Lambert, C. J. Eiting, C. J. Collins, T. Li, S Wang, B. Yang, A. L. Beck, R. D. Dupuis, and J. C. Campbell, "GaN avalanche photodiodes," Appl. Phys. Lett. vol. 76 (7), pp. 924-926, 2000

6. R. J. McIntyre, "Multiplication noise in uniform avalanche diodes,' IEEE Trans. Electron Devices. ED-13, pp. 164-168, 1966.

7. R. J. McIntyre, "The distribution of gains in uniformly multiplying avalanche photodiodes: Theory," IEEE Trans. Electron Devices. ED-19, pp. 703-713, 1972. 
8. I. H. Oğuzman, E. Bellotti, and K. F. Brennan, "Theory of hole initiated impact ionization in bulk zincblende and wurtzite GaN," J. Appl. Phys. vol. 81, pp. 7827, 1997.

9. S. Chen and G. Wang, "High-field properties of carrier transport in bulk wurtzite GaN: A Monte Carlo perspective," J. Appl. Phys. vol. 103, pp. 023703, 2008.

10. F. Bertazzi, M. Moresco, and E. Bellotti, "Theory of high field carrier transport and impact ionization in wurtzite GaN. Part I: A full band Monte Carlo model," J. Appl. Phys. vol. 106, pp. 063718, 2009.

11. F. M. A. El-Ela and A. Z. Mohamed, "Electron Transport Characteristics of Wurtzite GaN," ISRN Condensed Matter Phys., vol. 2013, pp. 654752

12. B. E. A. Saleh and M. C. Teich, Semiconductor Photon Detectors. In: Fundamentals of Photonics, John Wiley \& Sons, 2007, pp. 748-800.

13. S. M. Sze and K. K. Ng, Physics of Semiconductor Devices, Wiley Interscience, 2007, pp. 683-693.

14. L. Ardaravicius, M. Ramonas, J. Liberis, O. Kiprijanovic, A. Matulionis, J. Xie, M. Wu, J. H. Leach, and H. Morkoc, "Electron Drift Velocity in Lattice-Matched AlInN/AIN/GaN Channel at High Electric Fields," J.Appl.Phys., vol. 106, no. 7, pp. 073708(5), 2009.

15. F. Schwierz, "An electron mobility model for wurtzite GaN," Solid-State Electron. Vol. 49 (6), pp. 889-895, 2005.

16. M. Farahmand, C. Garetto, E. Bellotti, K. F. Brennan, M. Goano, E. Ghillino, G. Ghione, J. D. Albrecht, and P. P. Ruden, "Monte Carlo Simulation of Electron Transport in the III-Nitride Wurtzite Phase Materials System: Binaries and Ternaries," IEEE Trans. Electron Devices, vol. 48(3), pp. 535, 2001.

17. J. M. Barker, R. Akis, T. J. Thornton, D. K. Ferry, and S. M. Goodnick, "High Field Transport Studies of GaN," Physica Status Solidi (a), vol. 190(1), pp. 39-41, 2002.

18. C. G. Rodrigues, Á. R. Vasconcellos, and R. Luzzi, "Theoretical calculations of nonlinear electronic transport behavior in III-nitrides: GaN and AlN," Physica Status Solidi (b), vol. 246(2), pp. 417-425, 2009.

\section{AUTHORS PROFILE}

Pei Ling Cheang received her M.EngSc. degree from the Multimedia University (MMU) and B.Sc. (Hons.) in Applied Physics from the University of Malaya (MU). She is currently pursuing $\mathrm{PhD}$ degree in Engineering in Multimedia University (MMU). She is a member of Malaysia of Institute Physics.

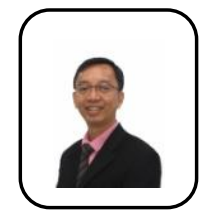

Dr. Eng Kiong Wong received his B.Eng. (Hons) degree in Electronic Engineering and $\mathrm{PhD}$ from the University of Western Australia (UWA), Perth, in 1995 and 2000 respectively. Since 1998, he has been attached to the Multimedia University (MMU) where he engaged in research in the field of optoelectronics, electronics, embedded system and robotics. He is a graduate member of the Board of Engineers Malaysia (BEM) and a graduate member of the Institution of Engineers Malaysia (IEM).

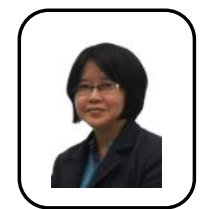

Dr. Lay Lian Teo received her $\mathrm{PhD}$ degree in Engineering from the Multimedia University (MMU), MSc. in Material Science and B.Sc. (Hons.) from the University of Malaya (MU). 